\title{
Holoprosencephaly and Dandy Walker Malformation: A Rare Association Presenting as Birth Asphyxia
}

\section{Rashmita Nayak ${ }^{1}$ and Suvendu Mohapatra ${ }^{2 *}$}

${ }^{1}$ Department of Pediatrics, S.C.B Medical College, Cuttack, Odisha, India

${ }^{2}$ Department of Radiology S.C.B Medical College, Cuttack, Odisha, India

${ }^{*}$ Corresponding author: Dr. Suvendu Mohapatra M.D, Senior Resident, Department Of Radiology, S.C.B Medical College \& Hospital, Cuttack, Odisha, 753007, India, Tel: +09861412800; Fax-+916742390674; E-mail: drsuvendumohapatra@gmail.com

Rec date: April 30, 2014; Acc date: June 03, 2014; Pub date: June 05, 2014

Copyright: (C) 2014 Nayak R, This is an open-access article distributed under the terms of the Creative Commons Attribution License, which permits unrestricted use, distribution, and reproduction in any medium, provided the original author and source are credited.

\begin{abstract}
Background: Holoprosencephaly a type of neuronal migration defect may present as birth asphyxia and is usually associated with other central nervous system and/ or systemic malformations. But its association with Dandy Walker cyst is rare.
\end{abstract}

Case characteristics: In a 6 month old male child presenting with global developmental delay since birth and refractory seizures with microcephaly and hypotonic extremities all attributed as birth asphyxia sequel neuroimaging revealed holoprosencephaly and dandy walker malformation, two major malformations in one child.

Observation: Till nine months child was considered to be post asphyxia sequel due to global developmental delay and seizures but only neuroimaging pointed towards the malformations. Computed tomography revealed presence of Dandy Walker malformation with semilobar holoprosencephaly which was the reason for the refractory seizures and thus had guarded prognosis.

Outcome: On three months follow up the child continued to have refractory seizures and delayed development.

Message: Central nervous system malformations may be symptomatic from birth and may present with poor apgar score at birth, refractory seizures and global developmental delay due to which they may be confused with birth asphyxia. Timely neuroimaging can make the diagnosis and thus the prognosis.

Keywords: Holoprosencephaly; Dandy Walker Malformation; Hypoxic ischemic encephalopathy

\section{Case Report}

A nine months old male child presented to us for the first time with history of refractory seizures beginning from second day of life. The child was born of normal vaginal delivery at term didn't cry immediately after birth requiring respiratory support in the form of bag and mask ventilation for few minutes due to cyanosis. The mother had no history of diabetes mellitus, hypertension, drug intake or irradiation during pregnancy with normal titers of toxoplasma, rubella, syphilis and hepatitis $B$. Birth weight was $3 \mathrm{~kg}$ and details of height and head circumference at birth was not available. The child developed seizures from the day two of life and required three anticonvulsants namely phenobarbitone, valparin and levetiracetam but still having intermittent tonic posturing. There was gross global developmental delay. At nine months the weight was $6 \mathrm{~kg}$ and head circumference was $36 \mathrm{~cm}$ i.e. child was having microcephaly. Generalized hypotonia with brisk deep tendon reflexes and extensor plantar reflex. Posterior fontanelle was slightly distended with normal tension in the anterior fontanelle. There was no morphological abnormality. Computed tomography was done which revealed two major and rare CNS malformations like coexistent Dandy walker malformation with semilobar holoprosencephaly. Features of classical
Dandy Walker malformation like hypoplastic cerebellar vermis and cephalad rotation of the vermian remnant with cystically dilatated fourth ventricle communicating with posterior fossa cyst, enlarged posterior fossa with torcular-lambdoid inversion that is torcular lying above the level of the lambdoid due to abnormally high tentorial insertion. Along with this associated features like monoventricle, fused thalami and incomplete separation of bilateral cerebral hemisphere, hypoplastic corpus callosum were seen. The child was managed conservatively and parents were explained about the prognosis (Figures 1-5).

\section{Discussion}

Holoprosencephaly is a neuronal migration disorder in which the prosencephalon (the forebrain of the embryo) fails to develop into two hemispheres and is usually associated with normal posterior fossa structures. Holoprosencephaly associated with Dandy walker cyst is rare. Till date only few cases have been reported [1-6]. Five of them had semilobar and two had alobar type of holoprosencephaly. None of them had any major facial or palatal malformation. All cases were complicated with hydrocephalus. In two of the cases ventriculoperitoneal shunting was done [3,5]. It seems obvious that holoprosencephaly is an organ formation abnormality due to disturbed diverticulation of prosencephalon at 5 to 6 weeks of gestation. 


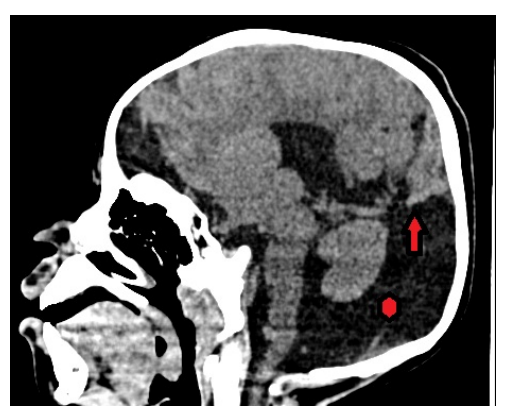

Figure 1: Sagittal CT scan in a 6 month old baby shows a large posterior fossa cyst (red hexagon) elevating the torcular herophili and sinus rectus (red arrow). The cerebellar hemispheres and brainstem are hypoplastic.

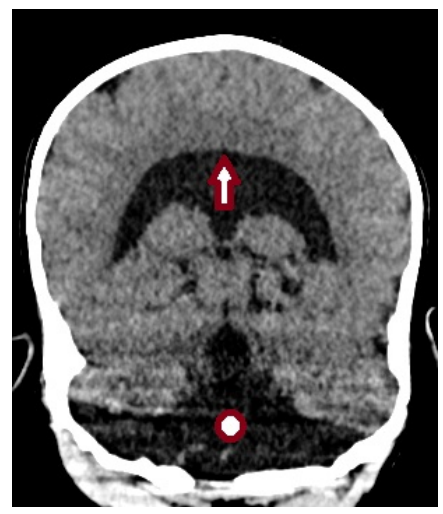

Figure 2: Coronal CT scan in a 6 month old baby shows semilobar holoprosencephaly with Horse shoe shaped monoventricle (red arrow). Basal ganglia and thalami are partially fused. Large posterior fossa cyst seen below (red circle).

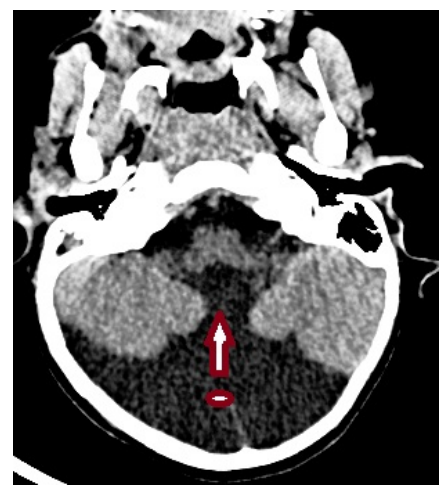

Figure 3: Axial CT scan in a 6 month old baby shows a large posterior fossa cyst communicating with fourth ventricle in dandy walker malformation.

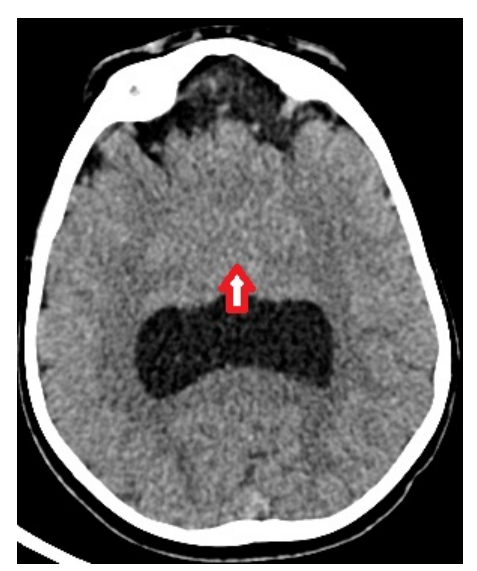

Figure 4: Axial CT scan in a 6 month old baby with semilobar holoprosencephaly shows fused thalami in midline.

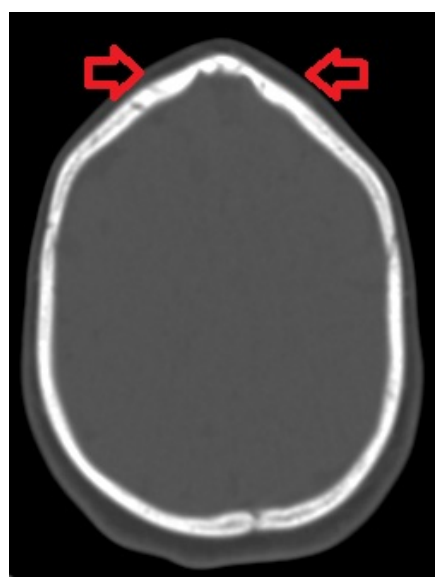

Figure 5: Axial CT scan in a 6 month old baby with semilobar holoprosencephaly with dandy walker malformation shows' lemon sign' of skull.

Tokimura et al. first described the mechanism of the coexistence of holoprosencephaly and Dandy walker cyst [5]. They postulated that these rare conditions are created by cerebrovascular disorders of the vertebrobasilar system at 4 to 5 weeks gestation. The vascular abnormality might result in central nervous system abnormalities. Cystic enlargement of the 4th ventricle and hypoplastic cerebellum are characteristic clinical manifestation of Dandy walker cyst. This mechanism is explained by disturbance of cerebrospinal fluid circulation from the rhombic roof at 5 to 6 weeks of gestation [7]. Dandy-Walker cyst-peritoneal shunt can be done in case of increased intracranial pressure. But in our case this was not so and so surgery was deferred with parents' consent.

\section{Conclusion}

From this it is concluded that central nervous system anomalies may present as poor apgar score at birth and subsequently global development delay. Timely diagnosis using advanced neuroimaging 
Citation: Nayak R, Mohapatra S (2014) Holoprosencephaly and Dandy Walker Malformation: A Rare Association Presenting as Birth Asphyxia. J

Page 3 of 3

can establish the diagnosis and thus prognosis. Even it can be detected antenataly in first and second trimester.

\section{References}

1. Hayashi T, Kuramoto S, Takagi S, Kojyo N (1980) Two cases of holoprosencephaly. Shoni No Noshinkei 5: 153-160.

2. Maeda T, Yokota H, Ogashiwa M, Takeuchi K (1984) Holoprosencephaly with Dandy walker cyst and aqueductal atresia. Shoni no Noshinkei 9: 83-89.

3. Matsushima T, Miyazaki M, Ohye C, Tajima S, Handa I (1984) Two cases of dysgenetic hydrocephaly. Shoni No Noshinkei 9: 281-286.
4. Osaka K, Matsumoto S (1978). Holoprosencephaly in neurosurgical practice. J Neurosurgery 48: 787-803.

5. Tokimura H, Kadota K, Uetsuhara K, Oda H, Todoroki K, et al. (1987) A case of holoprosencephaly accompanied with dysgenesis of cerebellum. Shoni No Noshinkei 12: 423-428.

6. Yasutaka K, Hiromi T, Tsutomu S, Koichi k, Tamutsu T, et al. (1990) Holoprosencephaly with dandy walker cyst. J Chid's Nerv Syst 6: 51-53.

7. Brodal A , Hauglie E (1959) Congenital hydrocephalus with defective development of cerebellar vermis. J Neurol Neurosurg Psychiatry 22: 99-108. 